\title{
ИССЛЕДОВАНИЕ ОСОБЕННОСТЕЙ ДЕФИЦИТА МЫШЕЧНОЙ СИЛЫ И ВЛИЯЮЩИХ НА НИХ ФАКТОРОВ У ПАЦИЕНТОВ С КОМПРЕССИОННЫМИ ПЕРЕЛОМАМИ ТЕЛ ПОЗВОНКОВ НА ФОНЕ СИСТЕМНОГО ОСТЕОПОРОЗА
}

\author{
С Марченкова Л.А. ${ }^{1}$, Макарова Е.В. ${ }^{1}$, Еремушкин М.А. ${ }^{1}$, Шакурова Л.Р. ${ }^{1}$, Стяжкина Е.М. ${ }^{1}$, \\ Чесникова Е.И. ${ }^{1}$, Разваляева Д.В. ${ }^{1}$, Котенко Н.В. ${ }^{1}$, Новиков А.В. ${ }^{2}$, Щедрина М.А. ${ }^{2}$
}

\section{${ }^{1}$ Национальный медицинский исследовательский центр реабилитации и курортологии (НМИЦ РК)}

Россия, 121099, г. Москва, ул. Новый Арбат, д. 32

\author{
${ }^{2}$ Приволжский исследовательский медицинский университет (ПИМУ) \\ Россия, 603155, г. Н. Новгород, Верхне-Волжская набережная, д. 18
}

\begin{abstract}
Цель: изучить степень снижения силы мышц спины при переломах позвонков (ПП) на фоне остеопороза (ОП), выделить ассоциированные с этим факторы и диагностические инструменты для оценки данных нарушений.

Материалы и методы. Исследуемую группу составили 60 человек (56 женщин, 4 мужчин) в возрасте $65,43 \pm 7,12$ года с остеопоротическими ПП. В группу сравнения вошли 30 человек (28 женщин, 2 мужчины) в воз-

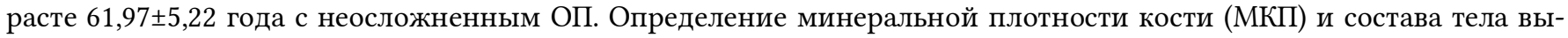
полнялось методом двухэнергетической абсорбциометрии, кондиционные способности оценивались с помощью функциональных тестов, сила мышц спины изучалась методом тензидинамометрии.
\end{abstract}

Результаты. У пациентов с остеопоротическими ПП в отличии от группы сравнения выявлен дефицит силы мышц сгибателей спины (СС) и разгибателей спины (РС) (p<0,001), при адекватной силе левых боковых сгибателей (ЛБС) и правых боковых сгибателей (ПБС). Выявлена обратная связь силы мышц от количества ПП (р<0,001), прямая связь с МПК. Сила РС зависела от минеральной массы скелета $(\mathrm{p}=0,03)$, сила СС - от массы мягких тканей $(\mathrm{p}=0,02)$, тощей массы $(\mathrm{p}=0,01)$ и минеральной массы скелета $(\mathrm{p}=0,003)$, сила ЛБС - от массы жировой ткани $(\mathrm{p}=0,03)$ и мягких тканей $(\mathrm{p}=0,03)$.

Заключение. ПП на фоне ОП ассоциируется со значимым снижением силы мышц, особенно в РС и СС, где формируется нефизиологическое распределение мышечной силы. У пациентов с ПП на фоне ОП мышечная масса сопоставима с лицами без переломов, но характрены низкие значения саркопенического индекса, массы и процентного содержания жировой ткани. Сила мышц спины у пациентов с ОП отрицательно зависит от количества ПП и положительно - от жировой массы, тощей массы и МПК.

Ключевые слова: остеопороз, саркопения, переломы позвонков, мышечная сила, состав тела.

Марченкова Лариса Александровна - канд. мед. наук, зав. отделением реабилитации пациентов с соматическими заболеваниями, зав. отделом соматической реабилитации, активного долголетия и репродуктивного здоровья, вед. науч. сотрудник, НМИЦ РК, г. Москва. ORCID iD: 0000-0003-1886-124X. E-mail: lr-march@rambler.ru

Макарова Екатерина Владимировна - науч. сотрудник отдела соматической реабилитации, активного долголетия и репродуктивного здоровья, НМИЦ РК, г. Москва. ORCID iD: 0000-0003-3767-8475. E-mail: rue-royal@inbox.ru (автор, ответственный за переписку)

Еремушкин Михаил Анатольевич - д-р мед. наук, профессор, зав. отделением ЛФК и клинической биомеханики, зав. отделом ортопедии, биомеханики, кинезитерапии и мануальной терапии, гл. науч. сотрудник, НМИЦ РК, г. Москва. E-mail: medmassage@mail.ru

Шакурова Лилия Рафильевна - мл. науч. сотрудник отдела соматической реабилитации, активного долголетия и репродуктивного здоровья, врач-эндокринолог отделения реабилитации пациентов с соматическими заболеваниями, НМИЦ РК, г. Москва. E-mail: lilya.shakurova@gmail.com

Стяжкина Елена Михайловна - канд. мед. наук, доцент, врач ЛФК отделения ЛФК и клинической биомеханики, вед. науч. сотрудник отдела ортопедии, биомеханики, кинезитерапии и мануальной терапии, НМИЦ РК, г. Москва. E-mail: styazelena@yandex.ru

Чесникова Екатерина Ивановна - врач ЛФК отделения ЛФК и клинической биомеханики, науч. сотрудник отдела ортопедии, биомеханики, кинезитерапии и мануальной терапии, НМИЦ РК, г. Москва. E-mail: ekaterina-bokova@mail.ru

Разваляева Дарья Владимировна - врач ЛФК отделения ЛФК и клинической биомеханики, НМИЦ РК, г. Москва.

Котенко Наталья Владимировна - канд. мед. наук, ст. науч. сотрудник отдела соматической реабилитации, активного долголетия и репродуктивного здоровья, врач-гинеколог, НМИЦ РК, г. Москва. E-mail: doktorkot@gmail.com

Новиков Александр Вульфович - д-р мед. наук, профессор, гл. науч. сотрудник Университетской клиники, ПИМУ, г. Н. Новгород. ORCID iD: 0000-0001-8099-3791. E-mail: novik2.55@mail.ru

Щедрина Марина Анатольевна - канд. мед. наук, ст. науч. сотрудник отделения реконструктивно-пластической хирургии и восстановительного лечения Университетской клиники, ПИМУ, г. Н. Новгород.

Неуклонный рост средней продолжительности жизни людей в России и во всем мире влечет за собой рост частоты заболеваний, ассоциированных с пожилым возрастом, одним из ко- торых является остеопороз - системное заболевание скелета, для которого характерно снижение прочности кости и повышение риска переломов [18]. Согласно данным Международного 
фонда остеопороза, каждые три секунды в мире происходит один перелом, ассоциированный с остеопорозом. В возрасте старше 50 лет каждая 3-я женщина и 5-й мужчина переносят хотя бы один остеопоротический перелом [3].

Патологические компрессионные переломы позвонков (ПП) - одно из наиболее частых и тяжелых осложнений остеопороза. В России ежегодно регистрируется 2 млн компрессионных ПП у женщин и 1 млн у мужчин [7]. Согласно эпидемиологическим исследованиям, проведенным в разных регионах страны, остеопоротические ПП встречаются у 7,2-12\% мужчин и $7-16 \%$ у женщин [7,3].

ПП у пациентов с ОП возникают в нижнегрудном и поясничном отделах позвоночника и в большинстве случаев сопровождаются выраженным болевым синдромом в спине, нарушением статики позвоночника, формированием патологического грудного гиперкифоза, ограничением подвижности и двигательной активности [21]. В связи с необходимостью формирования эффективных реабилитационных программ для таких пациентов, представляет интерес изучение особенностей нарушения функции мышц спины у пациентов с остеопорозом, перенесших ПП. Исследовательская гипотеза предполагает, что такая травма должна приводить к слабости мышц, мышечной дисфункции и кондиционным (базовым двигательным) нарушениям.

В этой связи также важным аспектом является выявление у этих больных клинических проявлений саркопении - возрастассоциированной потери массы и силы скелетной мускулатуры, которая является важным фактором риска новых переломов, а кроме того, может замедлять и снижать эффективность реабилитационных мероприятий [2].

Целью данного исследования было изучение специфических особенностей и степени снижения силы мышц спины при ПП на фоне системного остеопороза, определение факторов, в том числе клинических проявлений саркопении, влияющих на эти изменения, и выделение наиболее информативных диагностических инструментов для оценки нарушений кондиционных способностей у таких пациентов.

Статья будет полезна специалистам, работающим в сфере реабилитации и восстановительного лечения, травматологам, эндокринологам, врачам лечебной физкультуры.

\section{МАТЕРИАЛЫ И МЕТОДЫ ИССЛЕДОВАНИЯ}

Проводилось одномоментное исследование поперечного среза в двух группах. Исследуемую группу формировали из мужчин и женщин в возрасте от 40 до 80 лет, с системным остеопорозом, осложненным как минимум одним патологическим компрессионным ПП по критериям H. Genant $[12,13]$ давностью не более 12 месяцев, подтвержденным рентгенологически, и с показателями минеральной плотности кости (МПК) по Т-критерию в поясничном отделе позвоночника или проксимальном отделе бедренной кости $\leq-2,0$ по данным двухэнергетической рентгеновской абсорбциометрии [18].

Критериями исключения были: отказ подписать информированное согласие на участие в исследовании, кахексия любого происхождения, заболевания или лекарственная терапия, отрицательно влияющие на двигательные способности и мышечную силу.

Группу сравнения формировали из пациентов без патологических переломов в анамнезе, сопоставимых по полу, возрасту, индексу массы тела (ИМТ) и показателям МПК (Т-критерий в исследуемых зонах скелета $\leq-2,0)$, в соотношении 1:2 по отношению к исследуемой группе.

Комплекс исследований в обеих группах включал:

1. Общеклинический осмотр и сбор анамнеза;

2. Расчет абсолютного 10-летнего риска переломов при помощи международного инструмента-расчета FRAX® [3];

3. Костную денситометрию на двухэнергетическом рентгеновском абсорбциометре Lunar Prodigy (производство General Electric) для определения уровня МПК в поясничном отделе позвоночника (L1-L4) и проксимальном отделе бедренной кости и оценки композитного состава тела в режиме «Все тело» (Body total) с pacчетом массы жировой ткани (г), тощей массы (г), минеральной массы скелета (г) и скелетномышечного индекса Баумгартена - индекса саркопении $\left(\kappa г / \mathbf{m}^{2}\right)$.

4. Тензодинамометрию на аппарате BackCheck (производство Dr. Wolff, Германия) с оценкой силы мышц сгибателей спины (СС), разгибателей спины (РС), левых боковых сгибателей (ЛБС) и правых боковых сгибателей (ПБС). Аппарат регистрирует силу, с которой работает (оказывает сопротивление) определенная исследуемая группа мышц. Кроме абсолютных значений силы мышц в кг, аппарат определяет степень дефицита мышечной силы в процентах, которая рассчитывается как разница между фактическими показателями силы и показателями, рекомендованными для определенного возраста, пола, роста и веса;

5. Функциональные тесты на оценку кондиционных способностей: тест «Встань и иди», десятиметровый тест ходьбы, тест на выносливость мышц спины и живота к статической 
нагрузке, тест на выносливость мышц спины и живота к динамической нагрузке [2].

Статистический анализ проводился в программе Microsoft Statistica 10.0 с использованием параметрических и непараметрических методов. Значения показателей приведены в виде среднего и стандартного отклонения $(\mathrm{M} \pm \mathrm{m})$ при правильном распределении или в виде медианы и 25-го и 75-го квартилей (Ме [25\%; 75\%]) при неправильном. Для попарных сравнений показателей в группах использовали $\mathrm{t}$-критерий Стьюдента или критерий Манна-Уитни с поправкой Бонферрони. Для выявления взаимосвязи двух показателей для конечных величин использовали метод корреляции Спирмена или гамма-корреляции. Критический уровень значимости при проверке статистических гипотез принимался равным 0,05.

\section{РЕЗУЛЬТАТЫ ИССЛЕДОВАНИЯ И ИХ ОБСУЖДЕНИЕ}

В исследование включены 90 пациентов. Исследуемую группу составили 60 человек (56 женщин, 4 мужчины), из которых 38,3\% пациентов имели один патологический ПП, 16,6\% - 2 ПП, у $20 \%$ - 3 ПП, а в $25,1 \%$ случаев рентгенологически были подтверждены переломы в грудном и поясничном отделах позвоночника в количестве от 4 до 9. В группу сравнения включили 30 человек (28 женщин, 2 мужчин). Группы не различались по возрастным характеристикам, весу, росту, ИМТ, а также показателям МПК поясничного сегмента позвоночника (табл. 1). Однако пациенты в исследуемой группе имели более высокий абсолютный риск развития новых переломов по FRAX ${ }^{\circledR}$ в сравнении

Таблица 1 Table 1

Базовые характеристики исследуемой группы и группы сравнения

Baseline data on the study group and control group

\begin{tabular}{|c|c|c|c|}
\hline $\begin{array}{c}\text { Показатели } \\
\text { Indices }\end{array}$ & $\begin{array}{l}\text { Исследуемая группа } \\
\text { Study group }\end{array}$ & $\begin{array}{l}\text { Группа сравнения } \\
\text { Control group }\end{array}$ & $\mathrm{P}$ \\
\hline $\begin{array}{l}\text { Мужчины / женщины } \\
\text { Male / female }\end{array}$ & $4 / 56$ & $2 / 28$ & \\
\hline $\begin{array}{l}\text { Возраст (годы) } \\
\text { Age (years) }\end{array}$ & $65.4 \pm 7.1$ & $61.9 \pm 5.2$ & 0.09 \\
\hline $\begin{array}{l}\text { Bec (кг) } \\
\text { Weight }(\mathrm{kg})\end{array}$ & $74.8 \pm 14.2$ & $76.2 \pm 11.0$ & 0.10 \\
\hline $\begin{array}{l}\text { Рост }(\mathrm{cm}) \\
\text { Height }(\mathrm{cm})\end{array}$ & $161.3 \pm 16.3$ & $162.9 \pm 14.8$ & 0.12 \\
\hline $\begin{array}{l}\operatorname{ИМТ~}\left(\kappa г / \mathrm{M}^{2}\right) \\
\operatorname{BMI}\left(\mathrm{kg} / \mathrm{m}^{2}\right)\end{array}$ & $26.7 \pm 4.3$ & $28.7 \pm 5.94$ & 0.09 \\
\hline $\begin{array}{l}\text { Длительность остеопороза (лет) } \\
\text { Osteoporosis durarion (age) }\end{array}$ & $3.2 \pm 8.1$ & $2.9 \pm 7.03$ & 0.2 \\
\hline $\begin{array}{l}\text { Длительность менопаузы } \\
\text { Postmenopause duration }\end{array}$ & $15.8 \pm 8.0$ & $14.1 \pm 5.7$ & 0.10 \\
\hline $\begin{array}{l}\text { Возраст менопаузы } \\
\text { Age of menopause }\end{array}$ & $49.5 \pm 4.4$ & $49.8 \pm 4.4$ & 0.07 \\
\hline $\begin{array}{l}\text { Риск остеопоротических переломов по FRAX (\%) } \\
\text { Fracture risk according to FRAX (\%) }\end{array}$ & $23.0[17.5 ; 28.0]$ & $13.0[9.8 ; 16.0]$ & 0.000 \\
\hline $\begin{array}{l}\text { Риск перелома бедра по FRAX (\%) } \\
\text { Hip fracture risk according to FRAX (\%) }\end{array}$ & $6.9[3.6 ; 9.3]$ & $2.1[1.5 ; 3.9]$ & 0.000 \\
\hline $\begin{array}{l}\text { BMD L1-L4 (г/см) } \\
\text { BMD L1-L4 (g/cm) }\end{array}$ & $0.859 \pm 0.13$ & $0.965 \pm 0.12$ & 0.08 \\
\hline $\begin{array}{l}\text { BMD левая шейка бедра (г/см) } \\
\text { BMD left hip (g/cm) }\end{array}$ & $0.73 \pm 0.09$ & $0.88 \pm 0.11$ & 0.001 \\
\hline
\end{tabular}


Показатели состава тела по данным денситометрического исследования по программе «Все тело» в исследуемой группе и группе сравнения

Body composition data according to densitometry "Total body" in the study group and control group

\begin{tabular}{|c|c|c|c|}
\hline $\begin{array}{l}\text { Показатели } \\
\text { Indices }\end{array}$ & $\begin{array}{l}\text { Исследуемая группа } \\
\text { Study group }\end{array}$ & $\begin{array}{l}\text { Группа сравнения } \\
\text { Control group }\end{array}$ & $\mathrm{P}$ \\
\hline $\begin{array}{l}\text { Индекс Баумгартена (кг/м2) } \\
\text { Baumgarten index (kg/m2) }\end{array}$ & $6.52 \pm 1.2$ & $7.5 \pm 2.0$ & 0.02 \\
\hline $\begin{array}{l}\text { Мacca тела }(г) \\
\text { Body mass }(\mathrm{g})\end{array}$ & $75129.6 \pm 12178.4$ & $77726.4 \pm 16138.6$ & 0.09 \\
\hline $\begin{array}{l}\text { Macca мягких тканей (г) } \\
\text { Soft tissues mass (g) }\end{array}$ & $73197.6 \pm 11826.8$ & $75376.7 \pm 11745.9$ & 0.08 \\
\hline $\begin{array}{l}\text { Процент жировой ткани (\%) } \\
\text { Body fat (\%) }\end{array}$ & $40.67 \pm 7.2$ & $47.05 \pm 9.1$ & 0.01 \\
\hline $\begin{array}{l}\text { Macca жировой ткани (г) } \\
\text { Body fat mass (g) }\end{array}$ & $29717.0 \pm 8367.4$ & $35464.1 \pm 9127.4$ & 0.01 \\
\hline $\begin{array}{l}\text { Tощая масса }(г) \\
\text { Lean mass }(\mathrm{g})\end{array}$ & $43479.0 \pm 5969.11$ & $39949.5 \pm 6282.0$ & 0.07 \\
\hline $\begin{array}{l}\text { Минеральная масса скелета (г) } \\
\text { Mineral bone mass (g) }\end{array}$ & $1931.9 \pm 351.56$ & $2349.6 \pm 432.5$ & 0.001 \\
\hline
\end{tabular}

с контрольной группой, как для всех остеопоротических переломов $(\mathrm{p}=0,000)$, так и для перелома бедренной кости $(\mathrm{p}=0,000)$, а также более низкую МПК в шейке левого бедра $(\mathrm{p}=0,001)$.

По данным анализа состава тела, пациенты исследуемой группы с патологическими ПП, характеризовались более низким скелетномышечным индексом Баумгартена $(\mathrm{p}=0,02)$, меньшей массой и процентом содержания жировой ткани $(\mathrm{p}=0,01$ и $\mathrm{p}=0,001$ соответственно), а также меньшей минеральной массой скелета $(\mathrm{p}=0,001)$ при сопоставимой тощей массе $(\mathrm{p}=0,07)$ (табл. 2).

У пациентов исследуемой группы с ПП выявлен значительный дефицит силы СС и РС, при относительно адекватной силе боковых мышц сгибателей туловища (ЛБС и ПБС). В группе сравнения сила всех исследуемых мышц практически соответствовала рекомендуемым значениям - различия между рекомендованными и фактическими показателями не были статистически значимыми (табл. 3, рис. 1).

Абсолютные показатели мышечной силы (в кг) для всех групп мышц были значимо ниже в исследуемой группе, чем в группе пациентов без остеопоретических ПП (табл. 3 , рис. 1).

По данным тензодинамометрии обнаружены различия в силовом соотношении мышц РС и СС: у лиц с компрессионными ПП сила РС $(15,64 \pm 9,8$ кг) соотносилась с силой СС
$(14,61 \pm 8,98$ кг) приблизительно, как 1:1, тогда как у лиц без ПП отношение силы РС $(27,73 \pm 9,9$ кг) к

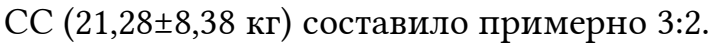

Как видно из табл. 4, не было выявлено статистически значимых различий между средними результатами функциональных тестов на оценку кондиционных двигательных способностей, полученных у пациентов в двух группах. Обращает на себя внимание, что показатели всех четырех тестов на выносливость к статической и динамической нагрузке у пациентов с ОП в обеих группах были одинаково критически низки, в сравнении с рекомендуемыми нормами выполнения данных тестов для лиц сопоставимого возраста [7].

Корреляционный анализ в исследуемой группе $(\mathrm{n}=60)$ выявил статистически значимую обратную зависимость силы всех исследуемых групп мышц спины от количества ПП ( $<<0,001)$, а также прямую связь со значениями МПК в поясничном отделе позвоночника (табл. 5). У пациентов с ПП на фоне ОП сила исследуемых мышц также положительно коррелировала с показателями состава тела. В частности, сила РС зависела от минеральной массы скелета $(\mathrm{p}=0,03)$, сила СС - от массы мягких тканей $(\mathrm{p}=0,02)$, тощей массы $(p=0,01)$ и минеральной массы скелета $(\mathrm{p}=0,003)$, тогда как сила ЛБС - от массы жировой ткани $(p=0,03)$ и мягких тканей $(p=0,03)$ (табл. 5). 
Показатели силы мышц туловища и дефицита мышечной силы в сравнении с рекомендованными значениями у пациентов с ОП

Trunk muscles strength and muscle strength deficiency in comparison with recommended values in patients with osteoporosis

\begin{tabular}{|l|c|c|c|}
\hline \multicolumn{1}{|c|}{\begin{tabular}{c}
\multicolumn{1}{|c|}{ Показатели } \\
Indices
\end{tabular}} & $\begin{array}{c}\text { Исследуемая группа } \\
\text { Study group }\end{array}$ & $\begin{array}{c}\text { Группа сравнения } \\
\text { Control group }\end{array}$ & P \\
\hline $\begin{array}{l}\text { Разгибатели спины (кг) } \\
\text { Trunk extensors (kg) }\end{array}$ & $15.64 \pm 9.8$ & $27.73 \pm 9.9$ & 0.000 \\
\hline $\begin{array}{l}\text { PC (\% дефицита силы) } \\
\text { ТЕ (strength deficiency \%) }\end{array}$ & $-40.93 \pm 39.19$ & $3.01 \pm 30.29$ & 0.000 \\
\hline $\begin{array}{l}\text { Сгибатели спины (кг) } \\
\text { Trunk flexors (kg) }\end{array}$ & $14.61 \pm 8.98$ & $21.28 \pm 8.38$ & 0.000 \\
\hline $\begin{array}{l}\text { СС (\% дефицита силы) } \\
\text { ТF (strength deficienсу \%) }\end{array}$ & $-18.12 \pm 46.6$ & $9.5 \pm 29.9$ & 0.006 \\
\hline $\begin{array}{l}\text { Наклоны туловища влево (кг) } \\
\text { Left lateral trunk fleхогs (kg) }\end{array}$ & $13.10 \pm 7.2$ & $24.06 \pm 8.9$ & 0.005 \\
\hline $\begin{array}{l}\text { ЛБС (\% дефицита силы) } \\
\text { LLTF (strength deficienсу \%) }\end{array}$ & $-8.2 \pm 23.55$ & $-8.5 \pm 10.84$ & 0.4 \\
\hline $\begin{array}{l}\text { Наклоны туловища вправо (кг) } \\
\text { Right lateral trunk flexors (kg) }\end{array}$ & $13.44 \pm 7.43$ & $24.26 \pm 7.65$ & 0.000 \\
\hline $\begin{array}{l}\text { ПБС (\% дефицита силы) } \\
\text { RLTF (strength deficiency \%) }\end{array}$ & $-8.42 \pm 20.81$ & $-6.6 \pm 9.0$ & 0.7 \\
\hline
\end{tabular}

Примечание: РС - разгибатели спины, СС - сгибатели спины, ЛБС - левые боковые сгибатели, ПБС правые боковые сгибатели.

Note: TE - trunk extensors, TE - trunk flexors, LLTF - left lateral trunk flexors, RLTF - right lateral trunk flexors.

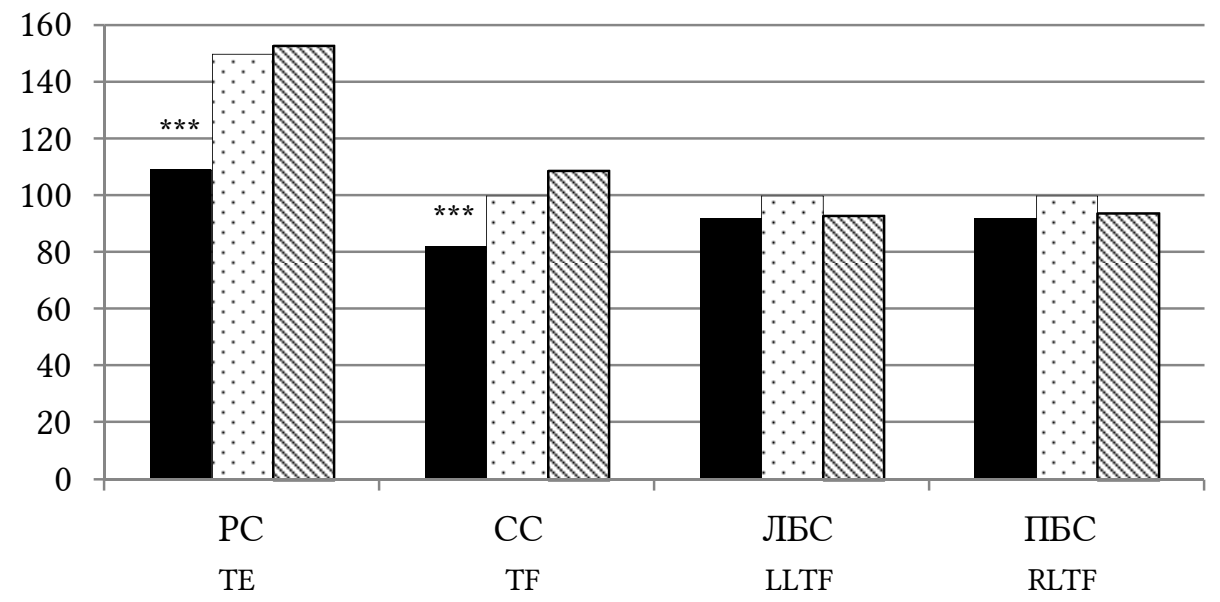
Исследуемая группа Study group

Рекомендованные значения

Recommended values

Группа сравнения
Control group

Рис. 1. Показатели мышечной силы (кг) в сравнении с рекомендуемыми значениями.

Примечание: РС - разгибатели спины, СС - сгибатели спины, ЛБС - левые боковые сгибатели, ПБС - правые боковые сгибатели; *** - различия статистически значимы между исследуемой группой и группой сравнения при значениях $\mathrm{p}<0,001$.

Fig. 1. Muscle strength data $(\mathrm{kg})$ in comparison with recommended values.

Note: TE - trunk extensors, TE - trunk flexors, LLTF - left lateral trunk flexors, RLTF - right lateral trunk flexors; ${ }^{* * *}$ - difference was statistically significant between study group and control group, $\mathrm{p}<0.001$. 
Результаты оценки кондиционных способностей в исследуемой группе и группе сравнения

Results of functional tests on conditional abilities in study group and control group

\begin{tabular}{|c|c|c|c|c|}
\hline $\begin{array}{l}\text { Показатели } \\
\text { Indices }\end{array}$ & $\begin{array}{l}\text { Нормы для 40-60 лет } \\
\text { Reference values } \\
\text { (for age 40-60) }\end{array}$ & $\begin{array}{l}\text { Исследуемая } \\
\text { группа } \\
\text { Study group }\end{array}$ & $\begin{array}{l}\text { Группа } \\
\text { cравнения } \\
\text { Control group }\end{array}$ & $\mathrm{P}$ \\
\hline $\begin{array}{l}\text { Тест «Встань и иди» (сек) } \\
\text { “Up and go" test (sec) }\end{array}$ & $\begin{array}{l}\text { до } 10 \\
\text { up to }\end{array}$ & $10.0[7.7 ; 13.0]$ & $9.0[8 ; 10]$ & 0.3 \\
\hline $\begin{array}{l}\text { Выносливость к статической } \\
\text { нагрузке мышц спины (сек) } \\
\text { Static back muscles endurance (sec) }\end{array}$ & $60-90$ & $0[0 ; 7]$ & $0[0 ; 30]$ & 0.29 \\
\hline $\begin{array}{l}\text { Выносливость к статической } \\
\text { нагрузке мышц живота (сек) } \\
\text { Static abdomen muscles endurance (sec) }\end{array}$ & $40-60$ & $0[0 ; 11]$ & $0[0 ; 0]$ & 0.18 \\
\hline $\begin{array}{l}\text { Выносливость к динамической } \\
\text { нагрузке мышц спины (раз) } \\
\text { Dynamic back muscles endurance (times) }\end{array}$ & 30 & $0[0 ; 5]$ & $0[0 ; 5]$ & 0.99 \\
\hline $\begin{array}{l}\text { Выносливость к динамической } \\
\text { нагрузке мышц живота (раз) } \\
\text { Dynamic abdomen muscles endurance (times) }\end{array}$ & 30 & $0[0 ; 5]$ & $0[0 ; 6]$ & 0.48 \\
\hline $\begin{array}{l}\text { Десятиметровый тест ходьбы (м/с) } \\
\text { 10-meters walking test (m/s) }\end{array}$ & $1.3-1.43$ & $1.6[1.2 ; 2.0]$ & $1.4[1.3 ; 1.7]$ & 0.37 \\
\hline
\end{tabular}

На показатели всех функциональных тестов по оценке кондиционных способностей статистически значимо влиял только возраст, за исключением теста «Встань и иди» (табл. 5). Однако не выявлено значимых связей результатов функциональных тестов с количеством переломов позвоночника, ИМТ, МПК, показателями состава тела или длительностью постменопаузы у женщин. Кроме того, обнаружена парадоксальная обратная зависимость между показателями теста на выносливость к статической физической нагрузке мышц спины и тощей массой (табл. 5).

Известно, что с возрастом у человека наблюдается постепенное ухудшение базовых двигательных способностей вследствие дегенеративных изменений в костно-мышечной и нервной системах $[17,20]$. Это проявляется значимым снижением выносливости, гибкости, силы и объема мышц [5]. Группой бельгийских ученых (Beaudart C. et al., 2016) в ходе исследования «SarcoPhAge» получены убедительные данные о связи развития инволютивных изменений в мышечной ткани (саркопении) с возрастом. При этом наличие низкой МПК и остеопороза напрямую значимо ассоциировалось с меньшим объемом скелетной мускулатуры и мышечной слабостью [10].

Известно, что саркопения чаще встречается у пожилых пациентов после какого-либо перенесенного перелома [18]. В целом данные исследований свидетельствуют о более низкой мышечной массе, мышечной силе, пониженной выносливости и гибкости у пациентов с остеопоротическими переломами в анамнезе, в сравнении с лицами того же возраста без остеопороза [11]. Большой вклад в развитие мышечной дисфункции у таких лиц вносит иммобилизация или недостаточная двигательная активность, поскольку в неработающих мышцах быстро запускаются инволютивные процессы [7].

Наше исследование было посвящено изучению проявлений саркопении и особенностей изменения мышечной силы фокусно у пациентов с остеопоротическими ПП. Полученные результаты показали, что мышечная масса у таких больных в целом сопоставима с лицами, страдающими неосложненным остеопорозом, однако выявлены меньшие значения массы и процентного содержания жировой ткани, а также саркопенического индекса, то есть более высокий риск развития саркопении. 
Корреляционные связи показателей мышечной силы по данным тензодинамометрии и функциональных тестов с возрастными, клиническими параметрами и составом тела

Correlations between muscle strength according to tension dynamometry and functional tests with age, clinical data and body composition

\begin{tabular}{|c|c|c|c|c|c|c|c|c|}
\hline $\begin{array}{l}\text { Параметры } \\
\text { Indices }\end{array}$ & 1 & 2 & 3 & 4 & 5 & 6 & 7 & 8 \\
\hline \multicolumn{9}{|c|}{$\begin{array}{c}\text { Тензодинамометрия (Back-Check Dr Wolff) } \\
\text { Tension dynamometry (Back-Check Dr Wolff) }\end{array}$} \\
\hline $\begin{array}{l}\mathrm{PC}(\kappa г) \\
\mathrm{TE}(\mathrm{kg})\end{array}$ & 0.01 & $-0.34^{* * *}$ & 0.02 & $0.39^{* *}$ & -0.29 & 0.12 & 0.17 & $0.31^{*}$ \\
\hline $\begin{array}{l}\mathrm{CC}(\kappa г) \\
\mathrm{TF}(\mathrm{kg})\end{array}$ & 0.08 & -0.27 *** & 0.1 & $0.48^{* *}$ & -0.22 & $0.34^{*}$ & $0.36^{* *}$ & $0.43^{* *}$ \\
\hline $\begin{array}{l}\text { ЛБС (кг) } \\
\operatorname{LLTF}(\mathrm{kg})\end{array}$ & 0.02 & -0.35 *** & 0.13 & $0.46^{* * *}$ & $0.32^{*}$ & $0.32^{*}$ & 0.09 & 0.22 \\
\hline $\begin{array}{l}\text { ПБС (кг) } \\
\operatorname{RLTF}(\mathrm{kg})\end{array}$ & -0.06 & $-0.32^{* * *}$ & -0.0 & $0.35^{*}$ & -0.15 & 0.1 & 0.11 & 0.25 \\
\hline \multicolumn{9}{|c|}{$\begin{array}{l}\text { Функциональные тесты } \\
\text { Functional tests }\end{array}$} \\
\hline $\begin{array}{l}\text { Тест «Встань и иди» (сек) } \\
\text { "Up and go" test (sec) }\end{array}$ & 0.23 & -0.07 & 0.001 & -0.19 & -0.013 & 0.09 & 0.16 & -0.009 \\
\hline $\begin{array}{l}\text { 10-метровый тест ходьбы (м/с) } \\
\text { 10-meters walking test }(\mathrm{m} / \mathrm{s})\end{array}$ & $0.27^{*}$ & 0.07 & 0.014 & -0.2 & -0.02 & -0.15 & 0.24 & -0.12 \\
\hline \multicolumn{9}{|c|}{$\begin{array}{c}\text { Выносливость к статической нагрузке } \\
\text { Static endurance }\end{array}$} \\
\hline $\begin{array}{l}\text { Мышцы спины (сек) } \\
\text { Back muscles (sec) }\end{array}$ & $-0.3 * *$ & 0.02 & -0.15 & -0.01 & -0.15 & -0.25 & $-0.31^{*}$ & 0.23 \\
\hline $\begin{array}{l}\text { Мышцы живота (сек) } \\
\text { Abdomen muscles (sec) }\end{array}$ & $-0.2^{*}$ & 0.12 & -0.13 & -0.14 & -0.15 & -0.12 & -0.03 & 0.005 \\
\hline \multicolumn{9}{|c|}{$\begin{array}{c}\text { Выносливость к динамической нагрузке } \\
\text { Dynamic endurance }\end{array}$} \\
\hline $\begin{array}{l}\text { Мышцы спины (раз) } \\
\text { Back muscles (times) }\end{array}$ & $-0.2^{*}$ & 0.05 & -0.08 & -0.000 & -0.08 & -0.16 & -0.25 & 0.21 \\
\hline $\begin{array}{l}\text { Мышцы живота (раз) } \\
\text { Abdomen muscles (times) }\end{array}$ & $-0.2^{* *}$ & -0.01 & -0.13 & -0.05 & -0.03 & -0.06 & -0.01 & 0.09 \\
\hline
\end{tabular}

Примечания: приведены коэффициенты корреляции Спирмена $\mathrm{r}$ или гамма-корреляции g. Столбцы: 1 - возраст (r), 2 - количество ПП (g), 3 - ИМТ (g), 4 - MПК L1-L4 (r), 5 - масса жировой ткани (r), 6 - масса мягких тканей (r), 7 - тощая масса (r), 8 - минеральная масса скелета (r). Различия статистически значимы между исследуемой группой и группой сравнения при значениях $\mathrm{p}^{*}<0,05,{ }^{* *}<0,01,{ }^{* * *}<0,001$. РС - разгибатели спины, СС - сгибатели спины, ЛБС - левые боковые сгибатели, ПБС - правые боковые сгибатели.

Notes: Spearman correlations (r) and gamma-correlations (g) were used. Columns: 1 - age (r), 2- number of vertebral fractures (g), 3 - BMI (g), 4 - bone mineral density L1-L4 (r), 5 - fat mass (r), 6 - soft tissues mass (r), 7 - lean mass (r), 8 - bone mineral mass (r). The difference was statistically significant between study group and control group, p values ${ }^{*}<0.05,{ }^{* *}<0.01,{ }^{* * *}<0.001$. TE - trunk extensors, TE - trunk flexors, LLTF - left lateral trunk flexors, RLTF - right lateral trunk flexors. 
Корреляционный анализ выявил, что показатели мышечной силы по данным тензодинамометрии обратно зависят от массы жировой и мышечной ткани. Доказано, что дефицит массы тела (в частности, объема жировой ткани) и саркопения, проявляющаяся уменьшением мышечной массы, являются факторами риска переломов в целом. Полученные нами данные позволяют заключить, что эти показатели можно считать факторами риска развития и патологических ПП у пациентов с остеопорозом.

Несмотря на практически равные значения мышечной массы, у пациентов с патологическими ПП уменьшается сила всех мышц туловища. Причем выявлена значимая обратная зависимость силы всех исследуемых мышц спины от количества перенесенных ПП. Самый выраженный дефицит силы отмечается в мышцах глубокой стабилизационной системы позвоночника, поддерживающих правильную осанку - РС и СС. Адекватное соотношение силы РС и СС предполагает преобладание функции разгибания над сгибанием. В норме соотношение PC:CC = 3:2 [1, 4], которое как раз и выявлено в группе сравнения у пациентов с ОП без патологических переломов. Для лиц с остеопоротическими ПП характерно нефизиологическое распределение силы мышц спины - РС:СС = 1:1, то есть имеет место не только количественный, но и качественный дефицит силы мышц спины.

Таким образом, развитие ПП на фоне системного остеопороза ассоциируется со значительным снижением силы всех мышц туловища, при этом наблюдается патологическая слабость мышц-экстензеров и гипертонус мышцфлексоров, что, очевидно, является причиной формирования патологического грудного гиперкифоза, ухудшения осанки и ограничения физического функционирования.

В нашем исследовании выявлена значимая прямая взаимосвязь силы всех мышц спины и МПК позвоночника, а также мышц сгибателей и разгибателей спины - с минеральной массой скелета. С одной стороны, можно сделать вывод, что величина потери МПК (то есть степень тяжести остеопороза) - еще один фактор, влияющий на степень потери мышечной силы у мужчин и женщин с ПП. С другой стороны, наоборот, в исследовании группы женщин 41-75 лет показана зависимость уровня МПК от исходного объема мышечной ткани, и продемонстрирован прирост костной массы на фоне увеличения объема и силы мышц [8]. Это подтверждается другими работами, где на примере молодых женщин отражено влияние мышечной силы на показатели МПК [9].

Известно, что низкий ИМТ (за счет низкой массы мышечной ткани) является предиктором развития остеопороза и переломов [14]. Однако, как показали наши результаты, величина ИМТ не влияет на силу и функцию мышц спины и результаты функциональных тестов у пациентов с остеопоротическими ПП.

В научных работах по оценке мышечной силы у пациентов с остеопорозом и пожилых лиц в целом часто используют функциональные тесты - шестиметровый и десятиметровый тесты ходьбы, тест «батарейки», тест «Встань и иди» и др. [15, 22]. При планировании дизайна исследования мы расширили традиционный спектр функциональных тестов на оценку кондиционных способностей, включив тесты на оценку мышц туловища к статической и динамической физической нагрузке, поскольку сила именно этих мышц параллельно оценивалась методом тензодинамометрии. Тем не менее не было выявлено каких-либо значимых отличий результатов функциональных тестов у пациентов с ПП по сравнению с лицами с остеопорозом без переломов. Показатели используемых тестов обратно зависели только от возраста пациента, но не были ассоциированы с числом ПП или уровнем МПК. При этом обращают на себя внимание крайне низкие по сравнению с нормативными (близкие к нулевым) показатели всех тестов на выносливость в обеих группах. Это может свидетельствовать либо об исходно завышенных нормах выполнения данных тестов [2], либо о низкой тренированности лиц среднего и старшего возраста в целом вследствие недостаточной приверженности культуре физических тренировок в повседневной жизни.

Таким образом, развитие патологических ПП на фоне ОП, как и заболевание само по себе, не влияют существенно на показатели функциональных тестов и поэтому последние, очевидно, мало информативны в оценке специфических изменений кондиционных способностей на фоне патологических ПП. Функциональные тесты обладают меньшей чувствительностью и валидностью (способностью выявлять именно тот признак, для которого они предназначены) по сравнению с тензодинамометрией мышц туловища по причине низкой точности измерений и присутствия субъективного фактора (оценка поводится врачом-исследователем). Важно, что показатели тензодинамометрии не зависят от возраста, а коррелируют только с выраженностью клинических проявлений остеопороза (уровнем МПК, количеством ПП) и саркопении (мышечная масса). Тензодинамометрия, очевидно, более предпочтительна в оценке мышечной силы и в сравнении с более распространенным и простым методом оценки функции мышц с помощью ручного динамо- 
метра [16], который позволяет судить лишь о состоянии мышц верхних конечностей.

Таким образом, на основании проведенных исследований можно сделать следующие выводы:

1. Развитие ПП на фоне системного остеопороза ассоциируется со значимым снижением силы всех мышц туловища. Самый выраженный дефицит силы отмечается в мышцах глубокой стабилизационной системы позвоночника РС и СС, где на фоне ПП формируется нефизиологическое распределение мышечной силы, равное 1:1, вместо 3:2, наблюдающееся в норме и у пациентов с неосложненным ОП.

2. У пациентов с ПП на фоне системного ОП мышечная масса сопоставима с лицами, страдающими неосложненным ОП, однако отмечены меньшие значения саркопенического индекса, массы и процентного содержания жировой ткани.

3. Сила мышц спины у пациентов с остеопорозом обратно зависит от количества ПП, прямо связана с жировой массой и объемом скелетной мускулатуры. Имеется прямая ассоциация МПК поясничного отдела позвоночника с силой мышц спины.

4. Не выявлено значимых отличий в результатах функциональных тестов у пациентов с ПП по сравнению с лицами с остеопорозом без переломов. Таким образом, в клинической практике, оценка силы мышц туловища с помощью тензодинамометрии более информативна, нежели стандартные функциональные тесты у лиц с ПП на фоне ОП.

5. Полученные данные стоит учитывать при разработке комплексов групповых и индивидуальных занятий лечебной физкультурой, подборе упражнений для занятий на тренажерах. Делать упор на тренировку мышечного корсета спины, особенно мышц разгибателей туловища.

\section{КОНФЛИКТ ИНТЕРЕСОВ}

Авторы декларируют отсутствие явных и потенциальных конфликтов интересов, связанных с публикацией статьи.

\section{ЛИЧНЫЙ ВКЛАД АВТОРОВ}

Марченкова Л.А. - разработка протокола исследования, анализ данных, написание текста статьи, формулирование выводов. Макарова Е.В. - реализация протокола клинического исследования, набор исследуемого материала, статистическая обработка, написание текста статьи. Еремушкин М.А., Новиков А.В. - научные консультанты. Шакурова Л.Р., Котенко Н.В., Чесникова Е.И., Стяжкина Е.М., Разваляева Д.В., Щедрина М.А. - реализация протокола клинического исследования, сбор данных.

\section{ИСТОЧНИКИ ФИНАНСИРОВАНИЯ}

Данная работа была выполнена без привлечения дополнительных финансовых средств.

\section{СООТВЕТСТВИЕ ПРИНЦИПАМ ЭТИКИ}

Протокол данного исследования одобрен на заседании локального этического комитета ФГБУ «НМИЦ РК» № 3 от 25.07.2016 года. Все участники исследования подписывали информированное согласие.

\section{ЛИТЕРАTУPA/REFERENCES}

1. Верхошанский Ю.В. Основы специиальной силовой подготовки в спорте. Изд. 3-е. Москва: Советский спорт; 2013. 216 с. [Verkhoshanskiy Yu.V. Osnovy spetsial'noy silovoy podgotovki $v$ sporte. $3^{\text {rd }}$ edition. Moscow: Sovetskiy sport; 2013. 216 p. (in Russ.)].

2. Еремушкин М.А. Двигательная активность и здоровье. От лечебной гимнастики до паркура. Москва: Спорт; 2016. 240 с. [Eremushkin M.A. Dvigatel'naya aktivnost' $i$ zdorov'ye. Ot lechebnoy gimnastiki do parkura. Moscow: Sport; 2016. 240 p.].

3. Лесняк О.М., Баранова И.А., Белова К.Ю., Гладкова Е.Н., Евстигнеева Л.П., Ершова О.Б., Каронова Т.Л., Кочиш А.Ю.и др. Остеопороз в Российской Федерации: эпидемиология, медикосоциальные и экономические аспекты проблемы (обзор литературы). Травматология и ортопедия Poсcuu. 2018; 24(1):155-168 [Lesnyak O.M., Baranova I.A., Belova K.Yu., Gladkova E.N., Evstigneeva L.P., Ershova O.B., Karonova T.L., Kochish A.Yu. et al. Osteoporosis in Russian Federation: epidemiology, socio-medical and economical aspects (review). Travmatologiya i ortopediya Rossii. 2018; 24(1):155-168 (in Russ.)].

4. Михайлофф П.Р., Громов В.А. Соотношение показателей развития силы мышц сгибателей и разгибателей туловища как фактор предотвращения боли в поясничном отделе позвоночника. Экстремальная деятельность человека. 2017; 43(2): 21-24 [Mikhayloff P.R., Gromov V.A. Relation of indicators between development of muscle power of flexor and extensor trunks, as a factor of pain prevention in the lumbar spine department. Ekstremal'naya deyatel'nost' cheloveka. 2017; 43(2):21-24 (in Russ.)].

5. Поворознюк В.В., Дзерович Н.И. Саркопения и возраст: обзор литературы и результаты собственных исследований. Боль. Суставы. Позвоночник. 2012;7(3):7-13 [Povoroznyuk V.V., Dzerovich N.I. Sarcopenia and age: literature review and results of own research. Bol'. Sustavy. Pozvonochnik. 2012; 7(3):7-13 (in Russ.)].

6. Российская ассоциация по остеопорозу. Остеопороз Диагностика, профилактика и лечение. Под ред. О.М. Лесняк, Л.И. Беневоленской. Изд. 2-е. Москва: ГЭОТАР-Медиа; 2009. 272 с. [Russian Association for osteoporosis. Osteoporoz Diagnostika, profilaktika i lecheniye. O.M. Lesnyak, L.I. Benevolenskaya, editors. $2^{\text {nd }}$ edition. Moskva: GEOTAR-Media; 2009. 272 p. (in Russ.)]. 
7. Хвостова С.А. Масса мышц, соединительной и жировой ткани в конечности после переломов. Современные проблемы науки и образования. 2011; 2:11 [Chvostova S.A. Mass of muscles, connective and adipose tissue in the limbs after fracture. Sovremennyye problemy nauki i obrazovaniya. 2011; 2: 11 (in Russ.)] URL: http://www.scienceeducation.ru/ru/article/view?id $=4580$.

8. Armamento-Villareal R., Aguirre L., Napoli N., Shah K., Hilton T., Sinacore D.R., Qualls C., Villareal D.T. Changes in thigh muscle volume predict bone mineral density response to lifestyle therapy in frail, obese older adults. Osteoporos Int. 2014; 25(2):551-558. DOI: 10.1007/s00198-013-2450-2.

9. Bayramoğlu M, Sözay S, Karataş M, Kilinç S. Relationships between muscle strength and bone mineral density of three body regions in sedentary postmenopausal women. Rheumatol Int. 2005; 25(7):513-517. DOI: $10.1007 / \mathrm{s} 00296-004-0475-8$.

10. Beaudart C., McCloskey E., Bruyère O., Cesari M., Rolland Y., Rizzoli R., Araujo de Carvalho I., Amuthavalli Thiyagarajan J. et al. Sarcopenia in daily practice: assessment and management. BMC Geriatr. 2016; 16(1):170. DOI: 10.1186/s12877-016-0349-4.

11. Cederholm T., Cruz-Jentoft A.J., Maggi S. Sarcopenia and fragility fractures. Eur J Phys Rehabil Med. 2013; 49:111-117.

12. Genant H.K., Jergas M., Palermo L., Nevitt M., Valentin R.S., Black D., Cummings S.R. Comparison of semiquantitative visual and quantitative morphometric assessment of prevalent and incident vertebral fractures in osteoporosis The Study of Osteoporotic Fractures Research Group. I Bone Miner Res. 1996; 11(7):984-996. DOI: 0.1002/jbmr.5650110716.

13. Genant H.K., Wu C.Y., van Kuijk C., Nevitt M.C. Vertebral fracture assessment using a semiquantitative technique. J Bone Miner Res. 1999; 8(9):1137-1148. DOI: $10.1002 /$ jbmr.5650080915.
14. Levinger I., Phu S., Duque, G. Sarcopenia and Osteoporotic Fractures. Clinic Rev Bone Miner Metab. 2016; 14(1):38-44. DOI: 10.1007/s12018-016-9204-6.

15. Lyons J.G., Heeren T., Stuver S.O., Fredman L. Assessing the Agreement Between 3-Meter and 6-Meter Walk Tests in 136 Community-Dwelling Older Adults. F Aging Health. 2015; 27(4):594-605. DOI: $10.1177 / 0898264314556987$.

16. McGrath R.P., Kraemer W.J., Vincent B.M., Hall O.T., Peterson M.D. Muscle Strength Is Protective Against Osteoporosis in an Ethnically Diverse Sample of Adults. F Strength Cond Res. 2017; 31(9):2586-2589. DOI: $10.1519 /$ JSC.0000000000002080.

17. Peterka R.J., Black F.O. Age-related changes in human posture control: sensory organization tests. f Vestib Res.1990-1991; 1(1):73-854.

18. Rikkonen T., Sirola J., Salovaara K., Tuppurainen M., Jurvelin J.S., Honkanen R., Kröger H. Muscle strength and body composition are clinical indicators of osteoporosis. Calcif Tissue Int. 2012; 91(2): 131-138. DOI: 10.1007/s00223-012-9618-1.

19. Tarantino U., Iolascon G., Cianferotti L., Masi L., Marcucci G., Giusti F., Marini F., Parri S. et al. Clinical guidelines for the prevention and treatment of osteoporosis: summary statements and recommendations from the Italian Society for Orthopaedics and Traumatology. F Orthop Traumatol. 2017; 18(S1):3-36. DOI: $10.1007 / \mathrm{s} 10195-017-0474-7$.

20. Volpi E., Nazemi R., Fujita S. Muscle tissue changes with aging. Curr Opin Clin Nutr Metab Care. 2004; 7(4):405-410.

21. WHO Scientific Group on the Burden of Musculoskeletal Conditions at the Start of the New Millennium. The burden of musculoskeletal conditions at the start of the new millennium. Geneva: World Health Organ Tech Rep; 2013. 231 p.

22. Zhou Z., Zheng L., Wei D., Ye M., Li X. Muscular strength measurements indicate bone mineral density loss in postmenopausal women. Clin Interv Aging. 2013; 8:1451-1459. DOI: 10.2147/CIA.S48447.

Поступила в редакцию 05.02.2019 Подписана в печать 20.06.2019

Для цитирования: Марченкова Л.А., Макарова Е.В., Еремушкин М.А., Шакурова Л.Р., Стяжкина Е.М., Чесникова Е.И., Разваляева Д.В., Котенко Н.В., Новиков А.В., Щедрина М.А. Исследование особенностей дефицита мышечной силы и влияющих на них факторов у пациентов с компрессионными переломами тел позвонков на фоне системного остеопороза. Курский научнопрактический вестник «Человек и его здоровье». 2019; (2):27-38. DOI: 10.21626/vestnik/2019-2/03. 


\title{
MUSCULAR AND STRENGTH DEFICIENCY IN PATIENTS \\ WITH OSTEOPOROTIC COMPRESSION VERTEBRAL FRACTURES: \\ OSTEOPOROSIS AS A RISK FACTOR FOR SARCOPENIA
}

\author{
○ Marchenkova L.A. ${ }^{1}$, Makarova E.V. ${ }^{1}$, Eryomushkin M.A. ${ }^{1}$, Shakurova L.R. ${ }^{1}$, Styazhkina E.M. ${ }^{1}$, \\ Chesnikova E.I. ${ }^{1}$, Razvalyaeva D.V. ${ }^{1}$, Kotenko N.V. ${ }^{1}$, Novikov A.V. ${ }^{2}$, Shchedrina M.A. ${ }^{2}$ \\ ${ }^{1}$ National Medical Research Center for Rehabilitation and Balneology (NMRC RB) \\ 32, Novyi Arbat, Moscow, 121099, Russian Federation \\ ${ }^{2}$ Privolzhsky Research Medical University (PRMU) \\ 18, Verkhne-Volzhskaya Embankment, N. Novgorod, 603155, Russian Federation
}

\begin{abstract}
Objective: to specify degree of back muscles strength deficiency in patients with osteoporosis (OP) and vertebral fractures (VF), to state associated risk factors and most informative diagnostic instruments.

Materials and methods. Study comprised 60 people aged $65.43 \pm 7.12$ with osteoporotic VF. The comparison group consisted of 30 people aged $61.97 \pm 5.22$ without VF. The assessment of bone mineral density (BMD) and body composition was performed by dual-energy absorptiometry, the conditioned abilities were estimated by functional tests, and back muscles strength was measured with tension dynamometry.

Results. Patients with VFs, unlike the comparison group, had a significant muscle strength deficiency in trunk flexors (TF) and extensors (TE) with an adequate function of the left lateral flexors (LLF) and right lateral flexors (RLF). Muscle strength negatively correlated with the number of VFs $(\mathrm{p}<0.001)$ and positively correlated with BMD strength of TE depended on bone mineral mass ( $\mathrm{p}=0.03)$, strength of TF - on soft tissues mass $(\mathrm{p}=0.02)$, lean mass $(\mathrm{p}=0.01)$ and bone mineral mass $(\mathrm{p}=0.003)$, strength of LLF - on fat mass $(\mathrm{p}=0.03)$ and soft tissues mass $(\mathrm{p}=0.03)$.

Conclusion. Osteoporotic VF are associated with a significant trunk muscles strength reduction, especially in TF and TE muscles, where non-physiological muscle strength distribution is found. In patients with osteoporotic VF, muscle mass is comparable to the group without VF, but lower values of sarcopenic index, fat mass and percentage are estimated. The strength of back muscles in patients with OP negatively correlates with number of VF, and positively correlates with fat mass, lean mass and BMD.
\end{abstract}

Keywords: osteoporosis, sarcopenia, vertebral fractures, muscular strength, body composition.

Marchenkova Larisa A. - PhD in Medicine, Head of Rehabilitation Department for Patients with Somatic Diseases, Head of Somatic Rehabilitation, Anti-Aging and Reproductive Health Department, Leading Researcher, NMRC RB, Moscow, Russian Federation. ORCID iD: 0000-0003-1886-124X. E-mail: lr-march@rambler.ru

Makarova Ekaterina V. - Researcher of Somatic Rehabilitation, Anti-Aging and Reproductive Health Department, NMRC RB, Moscow, Russian Federation. ORCID iD: 0000-0003-3767-8475. E-mail: rue-royal@inbox.ru (correspondence author)

Eryomushkin Mikhail A. - Doctor in Medicine, Professor, Head of Physical Therapy and Clinical Biomechanics Department, Head of Orthopedics, Biomechanics, Kinesiotherapy and Manual Therapy Department, Chief Researcher, NMRC RB, Moscow, Russian Federation. E-mail: medmassage@mail.ru

Shakurova Liliya R. - Junior Researcher of Somatic Rehabilitation, Anti-Aging and Reproductive Health Department, Endocrinologist of Rehabilitation Department for Patients with Somatic Diseases, NMRC RB, Moscow, Russian Federation. E-mail: lilya.shakurova@gmail.com

Styazkina Elena M. - PhD in Medicine, Associate Professor, Physical Therapy Physician of Physical Therapy and Clinical Biomechanics Department, Leading Researcher of Orthopedics, Biomechanics, Kinesiotherapy and Manual Therapy Department, NMRC RB, Moscow, Russian Federation. E-mail: styazelena@yandex.ru

Chesnikova Ekaterina I. - Physical Therapy Physician of Physical Therapy and Clinical Biomechanics Department, Researcher of Orthopedics, Biomechanics, Kinesiotherapy and Manual Therapy Department, NMRC RB, Moscow, Russian Federation. E-mail: ekaterinabokova@mail.ru

Razvalyaeva Darya V. - Physical Therapy Physician of Physical Therapy and Clinical Biomechanics Department, NMRC RB, Moscow, Russian Federation.

Kotenko Natalia V. - PhD in Medicine, Senior Researcher of Somatic Rehabilitation, Anti-Aging and Reproductive Health Department, Gynecologist, NMRC RB, Moscow, Russian Federation. E-mail: doktorkot@gmail.com

Novikov Alexander W. - Doctor in Medicine, Professor, Chief Researcher of University Clinic, PRMU, N. Novgorod, Russian Federation. ORCID iD: 0000-0001-8099-3791. E-mail: novik2.55@mail.ru

Shchedrina Marina A. - PhD in Medicine, Senior Rresearcher of Department of Reconstructive Plastic Surgery and Restorative Treatment of University Hospital, PRMU, N. Novgorod, Russian Federation.

\section{CONFLICT OF INTEREST}

The authors declare the absence of obvious and potential conflicts of interest related to the publication of this article.

\section{SOURCE OF FINANCING}

This work was carried out without additional funding.

\section{CONFORMITY WITH THE PRINCIPLES OF ETHICS}

The study protocol was approved at a meeting of the local ethics committee of FSBI "National Medical Research Center for Rehabilitation and Balneology" of Ministry of Health of Russia No. 3 of 25.07.2016. All participants of the study signed an informed consent.

\section{AUTHORS CONTRIBUTION}

Marchenkova L.A. - development of research protocol, data analysis, writing the text of the article, formulation of conclu- 
sions. Makarova E.V. - implementation of the clinical trial protocol, a set of materials under study, statistical processing, writing the text of the article. Eryomushkin M.A., Novikov A.V. - scientific consultants. Shakurova L.R., Kotenko N.V., Chesnikova E.I., Styazkina E.M., Razvalyaeva D.V., Shchedrina M.A. implementation of the clinical trial protocol, data collection.

For citation: Marchenkova L.A., Makarova E.V., Eryomushkin M.A., Shakurova L.R., Styazhkina E.M., Chesnikova E.I., Razvalyaeva D.V., Kotenko N.V., Novikov A.V., Shchedrina M.A. Muscular and strength deficiency in patients with osteoporotic compression vertebral fractures: osteoporosis as a risk factor for sarcopenia. Kursk Scientific and Practical Bulletin "Man and His Health". 2019;(2):27-38. DOI: $10.21626 /$ vestnik/2019-2/03. 